\title{
Lamarck et Jean-Jacques Rousseau
}

Par Jacques Roger

Jean-Baptiste de Monet de Lamarck (1744-1829) est universellement connu comme l'auteur de la première théorie de l'évolution des êtres vivants. Rien, dans la tradition familiale, ne conduisait ce petit noble picard à une carrière de naturaliste. Destiné à la prêtrise, il y échappa grâce à la mort de son père et réalisa sa vocation première, qui était d'être soldat. Son hérö̈sme obstiné lui valut d'être promu officier sur le champ de bataille, mais la fin de la Guerre de Sept Ans le contraignit bientôt à l'oisiveté de la vie de garnison. Il commença alors à herboriser, et les historiens attribuent souvent le choix de ce passetemps à l'influence de Rousseau, qui avait mis la botanique à la mode. Quoi qu'il en soit, Lamarck, forcé de quitter l'armée pour raisons de santé, se consacra à la botanique. Il semble que, installé à Paris, il s'en alla herboriser avec le Promeneur solitaire. Mais ses connaissances en botanique doivent beaucoup à Bernard de Jussieu qui enseignait au Jardin du Roi. La première œuvre scientifique de Lamarck, c'est en tout cas une Flore françoise (1778) qui lui valut la protection de Buffon et l'entrée à l'Académie des Sciences. En 1793, il prit une part active à la création du Muséum national d'Histoire naturelle, où il devint Professeur et où il enseigna avec succès aussi longtemps que ses forces le lui permirent. Membre de l'Académie Royale des Sciences, puis de la Première Classe de l'Institut national créé par la Révolution, Lamarck a fait partie toute sa vie de ce que l'on peut appeler la «science officielle», celle que consacrent les institutions d'Etat.

Derrière cette façade, cependant, se cache une personnalité moins conventionnelle et une vie difficile, qui, par certains aspects, et peut-être surtout par la manière dont elle a été vécue, n'est pas sans évoquer la vie de Jean-Jacques Rousseau. Avant d'avoir un petit poste au Jardin du Roi après la mort de Buffon, Lamarck avait été chargé de servir de mentor au fils du grand naturaliste, qui n'avait pas le génie de son père. Il l'avait escorté à travers l'Europe et avait dû supporter son arrogance et ses mauvaises plaisanteries. Situation typique du «précepteur», à la fois protégé et humilié, et d'autant plus ressentie peut-être que le Chevalier de Lamarck devait se sentir déclassé, et pouvait penser que sa noblesse était plus ancienne et plus militaire que celle du Comte de Buffon, ce bourgeois parvenu. Par la suite, 
jusqu'au professorat de 1793, et même au-delà, sa situation financière ne fut jamais brillante et il s'en plaignit souvent. Est-ce pour celà qu'il attendit, pour épouser Marianne Rosalie de la Porte, qui lui avait donné six enfants, qu'elle fût sur son lit de mort? Ce comportement était en tout cas peu conventionnel, même si Marianne Rosalie n'avait, semble-t-il, rien de commun avec Thérèse.

Si la vie privée de Lamarck ne fut jamais facile, sa vie professionnelle ne le fut pas non plus. En 1778, il avait rêvé d'exposer, dans le Discours Préliminaire de la Flore françoise, ses idées sur la nature, la vie, l'origine des minéraux et les véritables principes sur lesquels devraient reposer la physique et la chimie (Lamarck 1794, II, 366). Il semble que Buffon intervint pour censurer ces beaux projets et, par l'intermédiaire de Daubenton, fit confier à l'abbée Haüy le soin de corriger le texte et de limiter les ambitions encyclopédiques de l'auteur. Et ce d'autant plus que Lamarck s'en prenait vivement à la chimie de Lavoisier. «Protégé» de Buffon, Lamarck ne pouvait que s'incliner. Ce qu'il fit. Il remercia même Haüy «des services qu'il (avait) bien voulu (lui) rendre dans cet ouvrage» (1778, II, iv). Moyennant quoi, la Flore françoise fut imprimé aux frais du Roi et Lamarck toucha les bénéfices de la vente...

Il ne se résigna pas cependant. Tout en continuant à faire de la botanique, il rédigea un gros manuscrit (ou peut-être se contenta de le retoucher) et, le 22 avril 1780, le soumit à l'Académie des Sciences pour obtenir le droit de l'imprimer. Selon l'usage, l'Académie nomma des commissaires pour examiner l'ouvrage, mais ceux-ci, nous dit Lamarck, «mirent des lenteurs énormes dans l'examen de cet ouvrage» et ne rendirent jamais leur rapport (1794, vij). Du moins ne l'avaient-ils pas rendu quand la Convention, en 1793, supprima l'Académie. Libéré du despotisme de cette institution d'Ancien Régime, Lamarck publia son texte en l'An II de la République, sous le titre de Recherches sur les causes des principaux faits physiques. Il le dédia «Au Peuple français» et, devenu le «Citoyen Lamarck», il en fit hommage à la Convention nationale le 30 Fructidor de la même année $(1796, \mathrm{I})$.

L'Académie des Sciences n'existait plus, mais la Convention créa l'Institut National, c'est-à-dire un nouveau corps de savants officiels, aussi jaloux de leurs droits et de leurs prérogatives que les anciens. Lamarck s'en aperçut bientôt. Poursuivant sa croisade contre la nouvelle chimie, et ses «méditations» sur les mécanismes vitaux, il entreprit la lecture, devant la Classe des Sciences de l'Institut national, d'une série de mémoires où il exposait ses idées, afin, nous dit-il, «que mes principes sur la physique et les nouvelles 
considérations que je présentais y fussent sévèrement examinées et discutées très en détail. Mais je fus bien trompé dans mon attente, et l'effet de mes lectures à la première Classe de l'Institut fut toute autre que je ne devais le penser.

Il importe sans doute très-peu au lecteur d'avoir connaissance de ce que j'ai essuyé à cette occasion; des mépris et des repoussemens odieux dont je fus accueilli de la part des membres prépondérans et intéressés de cette classe; enfin, du refus constant que firent les chimistes de discuter aucune des questions présentées dans mes Mémoires, quoique la plupart fussent trèsneuves.

Ce que je dirai à cet égard, c'est que m'appercevant que mes lectures, dont on interrompait sans cesse le fil dans les séances, sous différens prétextes, paraissaient fatiguer plusieurs de mes collègues et leur être désagréables (...) je cessai tout-à-fait de solliciter la continuation de mes lectures; je n'achevai pas même celle du quatrième mémoire, qui a été commencée» (1797, 409-410).

Il ne restait à Lamarck qu'à publier ses mémoires, ce qu'il fit, en s'adresssant «à la portion du public non prévenue et non intéressée» ( Ibid.) Ses œuvres suivantes, celles auxquelles il tenait le plus, ne reçurent pas un accueil plus chaleureux. On ne les critique pas, ou peu: on se contenta de ne pas en parler. Peu à peu, Lamarck se mit à cultiver cette image du génie méconnu, travaillant dans la solitude, image que devait confirmer le désastreux éloge funèbre prononcé par son vieil ennemi Cuvier. «La postérité vous vengera, mon père», disait la fille de Lamarck. Elle le vengea en effet, au moins en ceci qu'elle reprit et amplifia l'image du génie solitaire. Mais Lamarck s'était déjà, pour ainsi dire, vengé de Cuvier, en expliquant que le penchant à dominer «est extrême dans l'homme à qui tout prospère (tandis que) la bonté, l'humanité, la modération, la sagesse même, ne se rencontrent guère que dans celui qui a beaucoup souffert de l'injustice des autres» (1820, 292). La philosophie, avait-il dit, doit conduire entre autres «à la résignation, et, s'il est possible, à l'impassibilité morale dans les souffrances, les revers, les injustices, les oppressions...» (Ibid., 291).

Lamarck fut-il, ou eut-il le sentiment d'être, le Jean-Jacques des sciences naturelles? Il ne l'a, à notre connaissance, jamais dit. Mais l'image du Promeneur solitaire apparaît bien en filigrane dans les épisodes de la vie de Lamarck que nous avons brièvement évoqués à travers ses propres textes: la conspiration du silence, le refus de discuter, «les mépris et les repoussemens odieux» et même presque l'appel «à tout Français qui aime encore la justice 
et la vérité». Et il est vrai que les savants officiels refusèrent de discuter la chimie de Lamarck et firent à peu près le silence sur sa théorie de l'évolution. Mais nous savons aussi que sa Flore française avait été un succès, que son œuvre de conchiliologie et de classification des invertébrés était universellement reconnue, même par Cuvier, que des savants plus jeunes, comme Latreille ou Etienne Geoffroy Saint-Hilaire, avaient beaucoup de respect pour lui, et qu'enfin, à côté de la «science officielle», il existait à l'époque un groupe influent de naturalistes non officiels, qui n'auraient pas demandé mieux que de voir Lamarck rejoindre leurs rangs, ce qu'il se refusa toujours à faire (Corsi 1979). Comme celle de Jean-Jacques, la solitude de Lamarck était fort entourée.

Mais la solitude est intérieure, et il importe peu d'être admiré, si ce n'est pas pour ce que l'on considère soi-même comme ses plus grandes qualités ou ses idées les plus originales. Nous ne saurons sans doute jamais si Lamarck a consciemment forgé son personnage sur celui de Rousseau, depuis le jeune déclassé réduit au préceptorat jusqu’au vieillard qui se croit «nul parmi les hommes» alors qu'il est partout connu. La chose, au moins, n'est pas impossible, et il y a entre eux d'indiscutables ressemblances de caractère et de destin.

Le second aspect de la présence de Rousseau chez Lamarck est d'un tout autre ordre, et sans doute beaucoup plus inattendu, puisqu'il s'agit de théorie chimique. Le mot «nature» est un de ceux qui reviennent le plus souvent sous la plume de Lamarck, mais il a chez lui un sens très précis, qui a pourtant beaucoup évolué depuis les premiers écrits jusqu'à sa philosophie dernière. Dans sa première œuvre, ces Recherches de 1794 qui avaient tant déplu aux Académiciens du Roi, la nature se ramène à la matière et à ses qualités. Traitant de chimie, Lamarck refuse à la nature le pouvoir de former aucun composé minéral ni aucun être vivant. Au contraire, la nature tend à «décomposer» les combinaisons chimiques. En un mot, «la nature ne forme rien, elle détruit toujours» (1794, II, 356. Souligné par L.). Affirmation surprenante, et qui rappelle le marquis de Sade et la Philosophie dans le boudoir plutôt que les idées de Jean-Jacques Rousseau.

Or c'est peut-être Rousseau qui est à l'origine de cette conception de la nature. Dire que la nature peut former des composés suppose, entre les éléments, des «affinités» qui les poussent à se combiner. Les chimistes admettent ces affinités, Lamarck les refuse. Car, explique-t-il, dans les composés solides il y a de très grandes quantités d'air et de feu. Dans ces composés, ils sont «dans un état de modification considérable (...), resserrés 
sous le plus petit volume possible en une quantité énorme et presque incroyable». Ils y sont donc "privés de leurs propriétés naturelles».

Ces combinaisons ne peuvent être spontanées, «car aucune sorte de matière ne peut avoir une tendance véritable à s'éloigner de son état naturel, à se détériorer, à se priver des facultés qui lui sont propres, à se modifier ellemême. Celà répugne et ne peut jamais être raisonnablement supposé. Il est évident, au contraire, que chaque élément, quel qu'il soit, doit tendre nécessairement à conserver toutes les qualités qui sont de son essence, et par conséquent à rester libre, et non à se modifier pour former un composé» (1794, II, 279-280).

Le composé chimique est donc pour l'élément ce que la société est pour l'homme selon Rousseau: une association où il a dû abdiquer ses facultés naturelles, et d'abord sa liberté, une situation de contrainte dans laquelle il est entré de force. Le feu libre (il s'agit ici de la «matière du feu», c'est-à-dire du «calorique») est expansible à l'infini; le «feu fixé» en combinaison est enfermé dans un volume dérisoire. On ne peut imaginer qu'il y soit entré de plein gré: «celà répugne», et il faut supposer une cause extérieure, en l'occurrence le «principe vital» capable de réduire l'élément à cette condition forcée, contraire à son «état naturel». Ainsi a-t-il fallu des circonstances extérieures pour amener les hommes à former les premières sociétés.

Mais en chimie, il n'est pas possible d'imaginer un «contrat social» qui permette aux éléments de former des combinaisons dans des conditions raisonnables. Donc, les matières entrées en combinaison «ont alors, par leur propre essence, une tendance manifeste à se dégager des corps qu'elles composent, et à perdre l'état de gêne où elles se trouvent»(Ibid., 281). Aussi tous les composés ont-ils une tendance à se détruire. Les éléments les plus expansifs, le feu, l'air, s'en échappent les premiers progressivement pour reprendre leur liberté. C'est ainsi que les composés organiques, que la vie a combinés de force, se «décomposent» dès que la vie les quitte, et forment tous les composés minéraux que l'on trouve dans la nature.

Il y a très loin de L'origine de l'inégalité à la physico-chimie de Lamarck, et pourtant le rapprochement nous semble légitime, dans la mesure où le vocabulaire lamarckien révèle une véritable mythologie de la liberté des éléments. Le caractère apparemment négatif que cette théorie confère à la nature ne tient qu'à nos conventions. Nous pensons spontanément que la composition chimique est une bonne chose, et nous la croyons naturelle, comme nous croyons depuis Aristote que la société est utile et que l'homme est naturellement un animal social. Si la liberté, au contraire, devient le bien 
suprême, la nature ne peut vouloir ni la combinaison ni la société. Dire que la nature «détruit toujours», celà revient à dire qu'elle libère, car seule la liberté est naturelle et apppartient à l'essence des êtres, hommes ou éléments. Liberté, c'est-à-dire solitude: le «feu libre» est seul dans son expansion infinie.

On sait que les théoriciens de la politique ont souvent pris la nature, telle qu'ils l'imaginaient, pour modèle des sociétés qu'ils décrivaient ou dont ils rêvaient. On sait aussi qu'à l'inverse, les savants ont souvent projeté sur la nature les structures et les valeurs de la société dans laquelle ils vivaient. Ici, nous avons une situation un peu différente: c'est une théorie politique qui sert de modèle à une interprétation de la nature. Il est vrai que cette théorie prétend se fonder sur la nature elle-même. Mais il s'agit de la nature de l'homme. Si Lamarck a pu prêter aux éléments cette tendance fondamentale à la liberté que Rousseau découvrait chez l'homme, c'est peut-être d'abord parce qu'il partageait intimement l'attitude de Rousseau en face de la société de son temps, attitude dans laquelle s'enracinait sa philosophie politique.

Jusqu'à présent nous n'avons pu faire état d'aucune référence explicite de Lamarck à Jean-Jacques Rousseau. Il n'en va pas de même pour le rapprochement que nous voulons tenter maintenant, et qui a déjà été rapidement évoqué par les historiens de Lamarck.

A partir de 1802, Lamarck est en possession d'une théorie générale des êtres vivants, théorie qu'il a été l'un des premiers à appeler «biologie». Cette théorie, il l'a exposée à plusieurs reprises, en la corrigeant ou en la complétant légèrement chaque fois: d'abord dans sa Philosophie zoologique en 1809, puis en 1815 dans sa longue Introduction à l'Histoire naturelles des animaux sans vertèbres, enfin, en 1820, dans le Système analytique des connaissances positives de l'homme. Contrairement à ce qu'une longue tradition historique peut donner à croire, l'objet essentiel de cette théorie n'est pas de démontrer et d'expliquer l'évolution des êtres vivants. C'est de montrer que ces êtres, et toutes les facultés qu'ils possèdent, sont vraiment des «productions de la nature».

Parmi ces facultés, certaines, comme la reproduction, sont universelles. D'autres, comme la sensibilité, exigent l'existence d'un système nerveux. D'autres enfin, comme l'intelligence, le jugement, la raison, n'existent que chez les animaux supérieurs, dont l'homme, parce qu'eux seuls possèdent l'organisation nécessaire à ces facultés. Mais toutes les facultés du vivant sont également «physiques», et Lamarck n'hésite pas à écrire: «L'idée est un phénomène organique» $(1820,290)$. 
On sait que, pour Lamarck, les êtres vivants ressentent des «besoins» correspondant à leurs conditions de vie, aux «circonstances» dans lesquelles ils se trouvent. Ces besoins sont des mouvements du «sentiment intérieur», c'est-à-dire du fluide nerveux. Les besoins habituellement ressentis créent des «penchants», que Lamarck énumère: penchant à la conservation, à la propagation de l'espèce, communs à tous les animaux sensibles. Ces penchants se traduisent par des actions, ce que nous appelons aujourd'hui «comportement» (1815, I, 263-267).

Chez les animaux intelligents, et particulièrement chez l'homme, le penchant à la conservation engendre trois penchants secondaires: tendance au bien-être, amour de soi-même, penchant à dominer ( Ibid., 267-71). Ainsi apparaît, mais en moins bonne compagnie, cet «amour de soi» que Rousseau tenait tant à distinguer de l'amour propre. Cependant, ces penchants peuvent agir, soit directement, sous le seul contrôle du «sentiment intérieur», soit sous le contrôle de «l'intelligence stimulée par le sentiment intérieur» ( Ibid., 272). Ils se manifestent alors de façon très différente, et la différence correspond très exactement à celle que Rousseau établit entre le comportement de l'homme de la nature et celui de l'homme de la société. Sous l'empire du seul sentiment intérieur, la tendance au bien-être ne pousse qu'à la nourriture, à la reproduction et au sommeil. Contrôlée par l'intelligence, elle engendre la chasse, la lâcheté, la ruse. Sous l'empire du sentiment intérieur, l'amour de soi-même peut pousser à s'emparer de la proie d'un autre animal ou à écarter un mâle concurrent d'une femelle convoitée, et le penchant à dominer poussera à se quereller, voire à tuer plus faible que soi; mais sous l'empire de l'intelligence, l'amour de soi engendrera l'attachement, la fidélité, la jalousie et la haine, et le penchant à dominer fera naître la fierté et le mépris pour les malheureux (Ibid., 272-75). L'état de nature selon Lamarck n'a rien d'idyllique, mais les vices et les vertus n'apparaissent que sous l'influence de l'intelligence.

Or on sait que, pour Lamarck, c'est le besoin ressenti qui pousse le sentiment intérieur à former ou à développer l'organe nécessaire à sa satisfaction. On sait aussi que l'usage développe un organe et que le nonusage l'atrophie. Le cerveau, et les facultés auxquelles il «donne lieu», comme dit Lamarck, n'échappent pas à cette règle, qui a été en fait la loi même de l'hominisation. Dans les pages bien connues de la Philosophie zoologique où il explique comment «une race de quadrumanes» aura pu donner naissance à l'espèce humaine, Lamarck précise que cette race, devenue dominante, aura pu «vivre par troupes nombreuses, se sera 
successivement créé des besoins nouveaux qui auront excité son industrie et perfectionné graduellement ses moyens et ses facultés« (1809, I, 351). L'intelligence, comme les autres facultés biologiques, se développe en fonction des besoins ressentis.

Ce qui est vrai des races est vrai des individus, au moins parmi les hommes. Il y a, dans toute société civilisée, une «échelle des différens degrés d'intelligence» $(1820,285)$. Cette inégalité intellectuelle n'a pas de base biologique: «Dira-t-on que le cerveau de cet homme de peine qui passe sa vie à maçonner des murs ou à porter des fardeaux, soit inférieur en composition ou en perfectionnement, à celui que possédèrent Montaigne, Bacon, Montesquieu, Fénelon, Voltaire, etc. malgré la différence infinie que l'on trouve entre l'intelligence dont ces hommes célèbres furent doués, et celle de l'homme du peuple que je viens de citer?» ( Ibid., 306-7). Non sans doute, et d'abord parce que cette forme d'inégalité n'existe pas en dehors des sociétés humaines. «Dans la nature, les animaux, vivant dans l'état sauvage, sont indépendants». Ils ont donc les mêmes facultés, et les seules différences que l'on trouve entre eux «tiennent à leur état physique, leur sexe, leur âge, leurs forces, leur état de santé, etc.».

Chez l'homme primitif, les différences intellectuelles sont très faibles. Mais la civilisation, en introduisant «le système des propriétés», a créé une inégalité croissante, réduisant «l'immense multitude à la pauvreté», la privant «des moyens de s'instruire» et l'obligeant «à des travaux grossiers et pénibles qui, en employant tout son temps, bornèrent considérablement ses idées».

«Ceux qui eurent plus d'activité, plus d'industrie, plus de courage», obtinrent dans la société «des situations progressivement plus avantageuses» qui leur permirent «de satisfaire à leurs besoins multipliés, d'augmenter leurs relations; d'acquérir plus d'instruction; d'agrandir davantage le cercle de leurs idées; quelquefois même, à l'aide de certaines circonstances, de multiplier et de varier considérablement ces idées». Car, «dans les situations les plus relevées de la société», les relations sociales se multiplient et, avec elles, les besoins et donc les idées. Parfois même, «une réunion de circonstances favorables» donne à l'intelligence «des développemens en quelque sorte extraordinaires» $(1820,281-4)$.

Telle est l'origine de cette «échelle des différens degrés d'intelligence» qui existe dans toute société civilisée, et met «l'immense majorité de ses habitants (...) à la merci d'une minorité dominante qui (...) mettra tout en usage pour en tirer le parti le plus convenable à ses intérêts» (Ibid., 288-9). 
Nous avons cité ici des textes du Système analytique de 1820, mais Lamarck avait déjà exprimé les mêmes idées, et parfois dans les mêmes termes, en 1815 dans l'Introduction à l'Histoire naturelle des animaux sans vertèbres et en 1817, dans divers articles du Nouveau Dictionnaire d'Histoire naturelle de Déterville. Car pour lui, ces considérations ne relèvent pas de la philosophie ni de la politique, mais simplement de la biologie. Science complète du vivant, la biologie doit expliquer aussi le comportement de tous les êtres vivants, y compris celui de l'homme en société. Mais le lecteur aura aisément reconnu le schéma historique du Discours sur l'origine de l'inégalité, l'opposition entre les inégalités naturelles, légères et sans conséquences, et les inégalités créées par la société, la peinture même d'une société divisée entre ceux qui ont tout et ceux qui n'ont rien, d'une société où l'injustice est inscrite dans les lois.

Dans le Système analytique, Lamarck fait explicitement un grand éloge de Jean-Jacques Rousseau, le «plus profond de nos moralistes». «Cet auteur, justement célèbre, revient à la nature dans ses ouvrages, et l'on voit qu'il avait le sentiment de l'importance de son étude, ainsi que celui des inconvénients, des dangers même de se mettre en contradiction avec ses lois. Plus passionné pour la nature qu'aucune des personnes qui me soient connues, les circonstances de sa vie ne lui permirent pas de la suivre dans sa marche, de bien saisir ses lois, de s'en instruire suffisamment». Ainsi s'est-il «douté de l'état de nos sciences, mais il les a condamnées d'une manière trop absolue». Malgré cette faiblesse, «les résultats auxquels il tendait partout, quoiqu'en indiquant des voies impropres, quelquefois contradictoires, sont toujours bons, justes et utiles à considérer» (1820, 94-5). Eloge nuancé, on le voit: Rousseau a eu un grand tort, celui de ne pas être biologiste et, en somme, de n'avoir été que philosophe. C'est précisément cette lacune que Lamarck prétend combler.

Cependant, l'enracinement biologique que Lamarck donne à son analyse de l'inégalité sociale soulève un certain nombre de problèmes. A première vue, celà signifie que les maux dont souffre la société tiennent à la nature même de l'homme, à ses «penchants», et non à «l'homme mal gouverné», comme le voulait Rousseau. Pourtant, Lamarck est très prudent sur ce point. Les mécanismes biologiques qu'il décrit et qui conduisent à l'abêtissement de la multitude sous l'empire des «circonstances» sociales, sont les mêmes qui, dans la nature, produisent l'atrophie définitive d'un organe chez un animal. Cependant Lamarck ne suggère jamais que l'abêtissement du peuple est définitif, que son intelligence est définitivement atrophiée. Il 
n'évoque pas non plus la possibilité de changer les circonstances sociales pour rendre le peuple intelligent. Mais sa théorie n'exclut pas ce changement et permet d'en attendre des conséquences heureuses. C'est ce qui autorisera les théoriciens du «lamarckisme social» sous la III $^{\mathrm{e}}$ République à fonder scientifiquement les grands espoirs qu'ils mettaient dans le développement de l'instruction publique et de l'hygiène. Lamarck leur avait explicitement tracé la voie, non pas en proposant des réformes, mais en décrivant la situation des hommes du peuple, «resserrés en général dans des lieux mal sains, ne respirant qu'un air vicié, irrégulièrement et mal nourris, se livrant à toutes sortes d'excès lorsqu'ils en trouvent l'occasion» et qui «voient, sans le remarquer, leur santé s'altérer, leur sang se vicier de mille manières, quantité de désordres divers se former dans leur organisation, enfin le germe d'un nombre considérable et toujours croissant de maladies différentes, et en quelque sorte endémiques, se transmettre et se perpétuer chez eux par la génération» (1820, 160-1). Tableau d'autant plus saisissant pour les législateurs de 1880 que, comme Rousseau, Lamarck fait de l'entassement des hommes dans les grandes villes une des tares du monde moderne, et semble ainsi décrire à l'avance le prolétariat urbain du XIX ${ }^{\mathrm{e}}$ siècle.

Cette possibilité ouverte à l'action réformatrice a beaucoup contribué, on le sait, à faire préférer, dans les années 1880 , la théorie lamarckienne de l'évolution à la théorie darwinienne, qui ne laissait espérer de progrès que dans l'action prolongée de la sélection naturelle et l'élimination des moins aptes. Mais on peut se demander si Darwin, après tout, n'avait pas été plus logique et conséquent que Lamarck. Car celui-ci, tout en soutenant, comme Darwin, que l'homme relevait de plein droit de la biologie, n'en avait pas moins évité de donner aux «circonstances» sociales des conséquences définitives. Peut-être pensait-il qu'elles n'avaient pas agi assez longtemps pour celà. Peut-être aussi n'a-t-il pas osé rendre tout espoir impossible.

Pourtant, ce n'est pas lui qui parle des réformes souhaitables. Il se contente de décrire ce qui est. Le seul conseil qu'il donne, c'est étudier la nature. C'est le sujet qui mérite le plus l'attention de l'homme car «presque tous ses maux, dans ce monde, lui viennent de ce qu'il le néglige (...) C'est uniquement de la connaissance de la nature, et de l'étude suivie de celles de ses lois qui sont relatives à son être physique, qu'il peut retirer pour sa conservation, pour son bien-être, et pour sa conduite, dans ses relations avec ses semblables, les seuls avantages réels qu'il puisse obtenir de l'observation» $(1820,55)$. On voit que, pour Lamarck, la nature n'est plus un modèle, une source d'inspiration pour l'action, une voix intérieure qui peut servir de 
guide. C'est l'ensemble des lois auxquelles nous sommes soumis et que nous ne pouvons espérer transformer. Il faut les connaître, pour mieux nous y adapter, mais non pas pour changer le monde, ou la société. Celle-ci n'est pas responsable de tout: «quelque changemens que la société ait fait éprouver à l'homme, quelque grandes que soient les améliorations qu'il en a retirées, et qui ne sont toujours que le propre d'un petit nombre, on le retrouve continuellement partout ce que la nature l'a fait, ayant les mêmes penchans, susceptible des mêmes passions, abusant ou opprimant ses semblables, se tourmentant lui-même» $(1820,161)$. Il n'est pas vrai que le mal soit seulement «de l'homme mal gouverné»: il est de l'homme lui-même.

Le biologiste ne peut donc pas partager les espoirs du philosophe. La conclusion que Lamarck tire de son tableau de la société, c'est qu'il faut y vivre au moins mal possible: «ce n'est que dans certaines situations, moyennes, entre la misère et la richesse ou les grandeurs, qu'on en voit jouir des douceurs d'une vie paisible et heureuse» (Ibid.). Avec, pour plus de sûreté, une solide morale stoïcienne qui permet la résignation et, si possible, l'impassibilité dans les revers et l'injustice des hommes.

Mais il n'y a pas que la biologie et la croyance au déterminisme universel qui séparent Lamarck de Rousseau: il y a aussi l'histoire. Rousseau écrivait au milieu du XVIII ${ }^{\mathrm{e}}$ siècle. Il vitupérait la société de son temps, mais il pouvait encore penser qu'une révolution était possible, même s'il n'a sans doute jamais prévu celle qui allait venir presque dix ans après sa mort. Lamarck a vécu la Révolution française. Selon toute vraisemblance, il y est entré avec joie et enthousiasme. Elle a changé son statut social, et transformé le petit «garde des herbiers» du Jardin du Roi en Professeur au Muséum, vivant au milieu de ses pairs, sans avoir à se soumettre à quelque «protecteur» que ce soit. Mais cette euphorie n'a pas duré. La nouvelle «science officielle» s'est révélée aussi despotique que l'ancienne. L'Empire a succédé à la République, et Napoléon, dans une scène célèbre, a traité le vieux Lamarck comme jamais Louis XVI n'aurait osé le faire. L'Introduction à l'Histoire naturelle des animaux sans vertèbres a été écrite à la fin de l'Empire; le Système analytique, au début de la Restauration. Qui pouvait croire alors qu'il suffisait d'une révolution pour changer la société? Ce que Lamarck retrouve, à la fin de sa vie, c'est l'attitude de Jean-Jacques à l'époque des Promenades, au moment où le vieux philosophe, abandonné de tous ou se croyant tel, se résigne à «être nul parmi les hommes» et à ne plus écrire que pour lui-même et pour la postérité. Ainsi l'histoire, autant que son destin personnel, a-t-elle pu dicter à Lamarck la résignation de ses derniers jours. 
C'est au lendemain de sa mort que la Révolution de 1830 donna aux Français le sentiment que l'histoire ne s'était pas définitivement arrêtée.

On a souvent voulu faire de Lamarck un simple héritier de la philosophie des Lumières. Sans entrer ici dans une discussion qui serait longue et hors de propos, qu'il nous suffise de dire que Lamarck est d'abord un homme de son temps, un contemporain de Laplace et de Cuvier, un des acteurs de cette seconde révolution scientifique qui ouvre le XIX ${ }^{\mathrm{e}}$ siècle. Sans doute est-il un héritier de la tradition de Locke et de Condillac, mais il est plus encore le contemporain de Cabanis, de Bichat, de Virey. S'il se distingue d'eux, c'est précisément dans la mesure où il rejette un aspect de la philosophie des Lumières, éminemment représenté par Diderot, et qui cherchait à faire de l'attraction et de la sensibilité des propriétés essentielles de la matière. Il est du côté de Haller contre Barthez, et son matérialisme doit plus à Boerhaave qu'à Cabanis.

Par contre, Rousseau semble avoir été toujours présent dans sa vie et dans son œuvre. Non pas, sans doute, dans le développement de ses idées proprement biologiques, dans les détails de ses opinions scientifiques. Il abandonnera même assez vite ses théories sur la «liberté» des éléments. En un sens, même, il renverse les schémas de Rousseau, dans la mesure où il montre que la nature, elle aussi, a une histoire, dans la mesure où il fait remonter le passé de l'homme bien au-delà d'un hypothétique «homme de la nature». Sur ce point précis, c'est Lacépède, plus que Lamarck, qui serait l'héritier de Rousseau (Barsanti 1979, 244-6). En outre, Lamarck ne semble pas faire de différence entre le processus qui a conduit du singe à l'homme primitif, et celui qui a mené de l'Homme primitif au civilisé. Le second devrait donc être aussi «naturel» que le premier.

Et pourtant, Lamarck ne peut s'empêcher d'opposer la nature à la société, et presque dans les mêmes termes que Rousseau. Ce n'est plus la même nature. Mais c'est toujours un guide à consulter, et peut-être un recours. Tout ce qu'il y a de contradictoire dans la pensée de Lamarck sur ce point permet de penser qu'il ne s'agit pas d'un emprunt purement intellectuel et théorique. Sans doute, beaucoup plus profondément, s'agit-il du sentiment d'une communauté de destin. 


\section{Bibliographie}

Barsanti (Giulio) 1979. Dalla storia naturale alla storia della natura. Milano, Feltrinelli. $8^{\circ}$, $261 \mathrm{p}$.

Burkhardt (Richard W.) 1977. The Spirit of System. Lamarck and Evolutionary Biology. Harvard University Press. $8^{\circ}, 285$ p.

Corsi (Pietro) 1983. Oltre il Mito. Lamarck e le scienze naturali del suo tempo. Bologna, Il Mulino. $8^{\circ}, 433 \mathrm{p}$.

Lamarck (Jean-Baptiste de Monet, chevalier de) 1778. Flore françoise. Imprimerie du Roi. 3 vol. $8^{\circ}$.

Id. 1786. Encyclopédie méthodique: botanique, tome II. Paris, Panckoucke. 4․․

Id. 1794. Recherches sur les causes des principaux faits physiques. Paris, Maradan. 2 vol. in- $8^{\circ}$.

Id. 1796. Réfutation de la théorie pneumatique. Paris, L'auteur. $8^{\circ} .484$ p.

Id. 1797. Mémoires de physique et d'histoire naturelle. Paris, L'auteur. $8^{\circ}, 420$ p.

Id. 1809. Philosophie zoologique. Paris, Dentu. 2 vol. in-8 ${ }^{\circ}$

Id. 1815. Histoire naturelle des animaux sans vertèbres, tome I. Paris, Verdière. $8^{\circ}$ (le tome VII paraîtra en 1822).

Id. 1820. Système analytique des connaissances positives de l'homme. Paris, L'auteur. $8^{\circ}, 364 \mathrm{p}$.

\section{Summary}

Lamarck (1744-1829) is well known as "the founder of evolutionary theory". An army officer converted to Botany, he probably herborized with the aging Jean-Jacques Rousseau, whose influence is to be found in his life and his writings. Though a member of the scientific establishment, Lamarck felt he was persecuted by his colleagues for his eccentric ideas on chemistry and evolution, as Rousseau had been by the Philosophes. Lamarck's chemical theory applies to chemical elements an idea of "natural freedom" clearly borrowed from Rousseau's political writings. In his latest works, he shows how biological processes multiply the effects of social inequality, explained according to Rousseau's views. Thus, his life and scientific writings bear unexpected testimony to Rousseau's lasting influence.

Jacques Roger

Université de Paris I

Panthéon

17, rue de la Sorbonne

Paris 